\title{
The prevalence of osteopenia and osteoporosis in out-patients above 40 years of a tertiary care hospital in Delhi
}

\author{
Ananya Sharma*, Alok Garg, Binit Singh
}

Department of Orthopaedics, Indira Gandhi ESI Hospital, New Delhi, Delhi, India

Received: 19 October 2021

Revised: 16 December 2021

Accepted: 17 December 2021

*Correspondence:

Dr. Ananya Sharma,

E-mail: ananyasharma@doctor.com

Copyright: ( ) the author(s), publisher and licensee Medip Academy. This is an open-access article distributed under the terms of the Creative Commons Attribution Non-Commercial License, which permits unrestricted non-commercial use, distribution, and reproduction in any medium, provided the original work is properly cited.

\begin{abstract}
Background: Osteoporosis is a common bone disorder among Indian population but is still underdiagnosed. Being a treatable disease, early diagnosis can significantly reduce osteoporotic fractures and other morbidities. Despite multiple studies globally regarding the prevalence of osteoporosis and osteopenia, the data about Indian population especially in males is limited. In this study, we aim to calculate the prevalence of osteoporosis in patients over 40 years of age attending the out-patients department of a tertiary care hospital in New Delhi.

Methods: A cross-sectional study was done including 231 patients (147 females and 84 males) over the age of 40 years. Calcaneal quantitative ultrasonography (QUS) was used to measure the bone mineral density and the results were divided into normal, osteopenic and osteoporotic groups on the based on equivalent heel $\mathrm{T}$ score.

Results: Of the total 231 patients, 78 patients had a normal bone mass density as measured by calcaneal QUS. 123 patients $(53.2 \%)$ were found to be osteopenic while 30 patients $(13.1 \%)$ were osteoporotic. Of the 147 females, 84 (57\%) were osteopenic while $27(18 \%)$ patients were found to be osteoporotic. Among the 84 males, 39 (46\%) were osteopenic and 3 (4\%) were found to be osteoporotic.

Conclusions: The prevalence of osteoporosis and osteopenia in outpatients over 40 years was high in our study and similar in values with other studies involving the Indian population. Hence, we recommend regular screening and early detection of osteoporosis to reduce the burden of the disease.
\end{abstract}

Keywords: Osteoporosis, Osteopenia, Bone mass density, QUS

\section{INTRODUCTION}

Osteoporosis is a disorder defined by low bone mass and microarchitectural deterioration of bone tissues leading to enhanced bone fragility and an increased risk of fracture. ${ }^{1}$ Bone loss is a normal process with ageing and occurs in both men and women after they have achieved a peak bone mass. This peak is usually achieved around the middle of the third decade. ${ }^{2}$ Osteoporosis usually presents as two types, Type 1 or the postmenopausal osteoporosis affecting women over 65 years of age and a Type 2 osteoporosis which is found in both men and women once the peak bone mass is achieved. ${ }^{3,4}$
According to the 2013 International Osteoporosis Foundation (IOF) Asian Audit, approximately 50 million people in India were either osteoporotic or had a low bone mass with $\mathrm{T}$ score less than $-1 .^{5}$ The Indian population also exhibits lower bone mass at a relatively younger age as compared to the global levels. ${ }^{6}$ In a 2011 study in Delhi, 1600 healthy adults with a mean age of 57.67 years were evaluated. $35.1 \%$ of the subjects were found to be osteoporotic while $49.5 \%$ were osteopenic. $^{7}$

Despite being a significant cause of morbidity, male osteoporosis is severely under diagnosed. Thus, most cases of male osteoporosis become evident only after a fracture 
has occurred. ${ }^{8}$ Moreover, most studies on osteoporosis have been focused on post-menopausal women leading to lesser data regarding the extent of the prevalence of osteoporosis in males. ${ }^{9,10}$

Osteoporosis is measured according to the World Health Organization (WHO) criteria which is a decrease in bone mineral density (BMD) of 2.5 standard deviations or more below that of the mean peak BMD of young adults when measured by dual-energy x-ray absorptiometry (DEXA). ${ }^{11}$ A BMD between -1 to -2.5 standard deviations is considered osteopenic. The DEXA scan is considered the gold standard in measuring bone mineral density but the high cost and difficulty in accessing the test render it unavailable to most Indian population. ${ }^{12,13}$

Calcaneal quantitative ultrasound (QUS) was first introduced in 1984 and has been used in multiple studies since then. ${ }^{14}$ When compared with the DEXA scan, QUS is portable, cheaper and does not emit any ionizing radiation. The QUS has proven to be a reliable and costeffective method as a preliminary tool for assessing bone mineral density. ${ }^{15,16}$

The aim of our study is to assess the prevalence of osteoporosis and osteopenia in a cross-sectional cohort of patients above 40 years of age presenting at the outpatient department of a tertiary health care centre in Delhi.

\section{METHODS}

This was a cross sectional study conducted in the Department of Orthopaedics, Indira Gandhi ESI Hospital, Jhilmil, New Delhi, India.

The duration of the study was from September 2021 to October 2021.

A total of 231 patients over the age of 40 years attending the Orthopedic OPD were selected for the study with prior consent. The study included 147 females and 84 males. The inclusion criteria were: age more than 40 years, both men and women were included, informed consent, no systemic disorder. The exclusion criteria were: age less than 40 years, history of calcaneal fractures, history of prolonged immobilization, use of pharmacological drugs affecting bone density, steroid use, major systemic disorders.

The bone mineral density (BMD) of all patients was measured using the calcaneal quantitative ultrasound (QUS). Even though, the WHO $\mathrm{T}$ score criteria for osteoporosis and osteopenia is defined for the DEXA scan, an equivalent heel $\mathrm{T}$ score has been proved to be an effective test to rule out or confirm osteoporosis, thereby avoiding a DEXA scan. ${ }^{17}$ Other studies have also reported the use of QUS as an effective screening method for osteoporosis using the same T score criteria. ${ }^{18,19}$
The BMD of all patients were measured using their right heel and an adjusted equivalent heel T score was recorded. The T-scores were divided into normal, osteopenia and osteoporosis, as per the WHO criteria with osteoporosis being $\mathrm{T}$ value less than 2.5 standard deviation, osteopenia being a decrease between 1 to 2.5 standard deviation and normal $(\leq-1.0 \mathrm{SD})$.

A statistical analysis was done and categorical variables were presented as percentages while the continuous variables were presented as mean. The purpose and importance of the study were explained and a written informed consent was obtained from each participant.

\section{RESULTS}

\section{Total patients and age wise distribution}

A total of 231 patients over the age of 40 years were included in the study including 147 females (63\%) and 84 males $(37 \%)$. The youngest patient was 40 years old and the eldest was 78 years with an average age of 49.14 years.

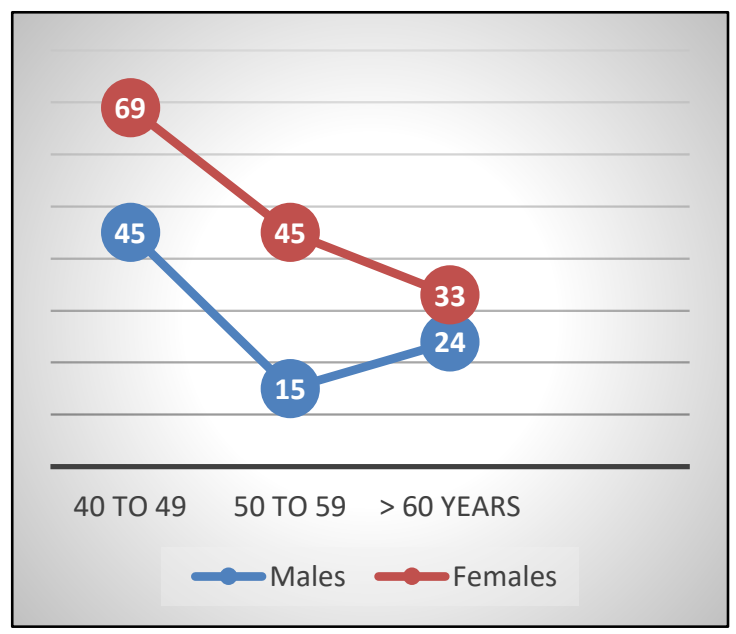

Figure 1: Age wise patient distribution.

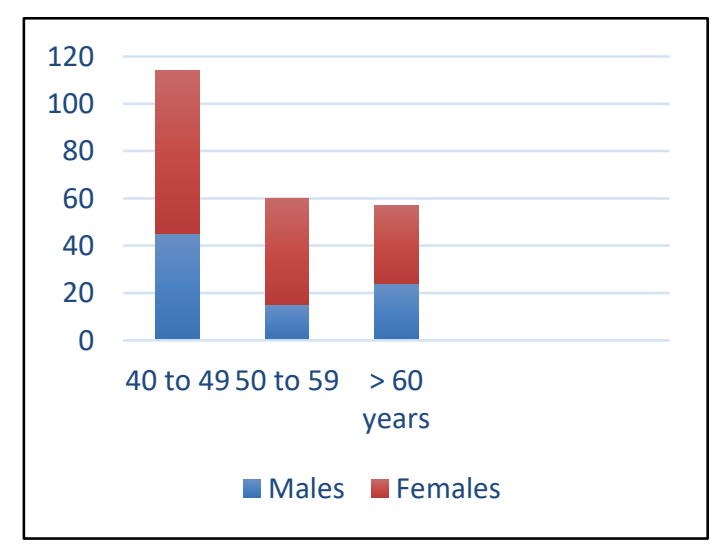

Figure 2: Age wise distribution.

They were further subdivided into 3 age groups - (I) Age 40 to 49, (II) Age 50 to 59, (III) Age 60 years and above. 
Of the 147 females, 69 were between 40 to 49 years, 45 were between 50 to 59 years and 33 were above 60 years. Among the males, 45 were between 40 to 49 years, 15 were between 50 to 59 years and 24 were over the age of 60 years.

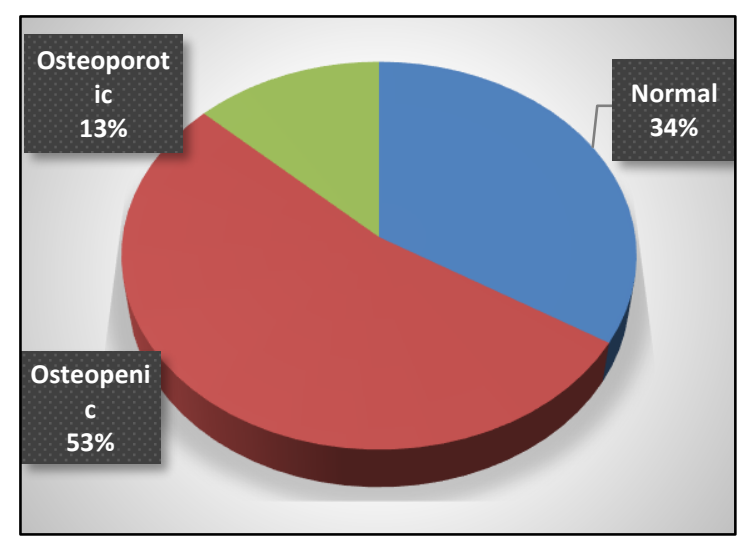

Figure 3: Prevalence of osteoporosis.

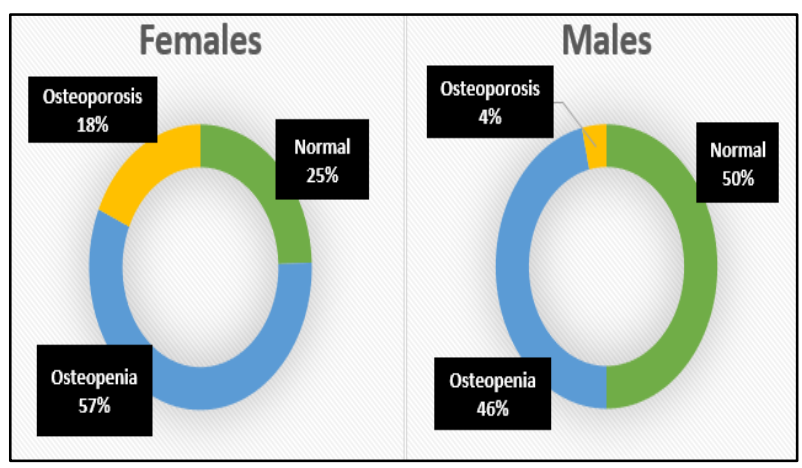

Figure 4: Sex distribution of prevalence of osteoporosis.

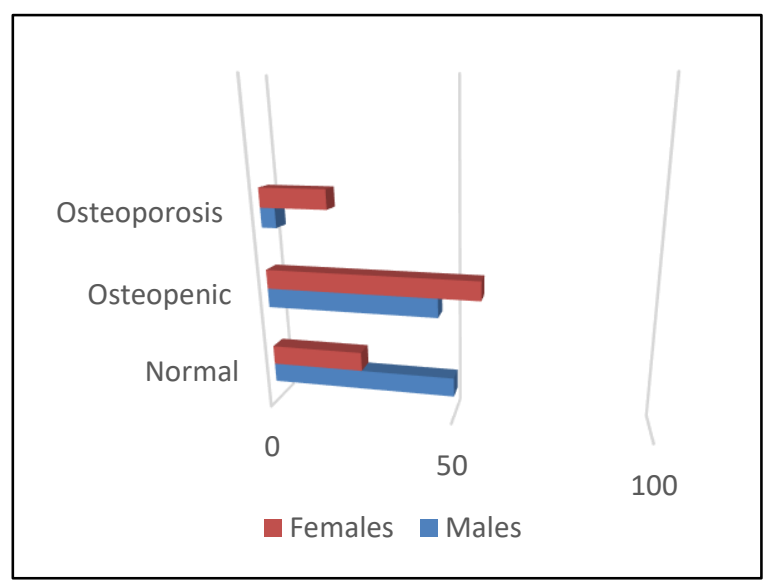

Figure 5: Prevalence of osteoporosis by percentage.

\section{Prevalence of osteoporosis and osteopenia}

Of the total 231 patients, 78 patients had a normal bone mass density as measured by calcaneal QUS. 123 patients were found to be osteopenic with the T Score between -1 and -2.5 SD while 30 patients were osteoporotic. Thus, $33.7 \%$ patients belonged to the normal group, $53.2 \%$ were osteopenic and $13.1 \%$ patients had osteoporosis.

\section{Sex distribution}

\section{Females}

Of the 147 females, 36 presented with a normal bone density, 84 were osteopenic while 27 patients were found to be osteoporotic. The overall prevalence of osteopenia was $57 \%$ and Osteoporosis was $18 \%$.

Males

Among the males, out of a total of 84, 42 had normal bone mass density, 39 were osteopenic and 3 were found to be osteoporotic. The prevalence of osteopenia was $46 \%$ and osteoporosis was $4 \%$.

\section{DISCUSSION}

The present study was done to assess the prevalence of osteopenia and osteoporosis in apparently healthy OPD patients from Delhi.

In women, the overall prevalence of osteopenia was 57\% and Osteoporosis was $18 \%$ according to our study. A similar study done by Marwaha et al for women over 50 years of age found $45 \%$ women to be osteopenic while $42 \%$ were diagnosed as osteoporotic. ${ }^{7}$ This increase in percentage could be attributed to a higher minimum age as an inclusion criterion. Another study by Gandhi et al of around 200 women from Mumbai over the age of 40 years found the prevalence of osteopenia in $34 \%$ and osteoporosis in $8 \%$ of the population while a study in Jammu using QUS put the values for the same as $37.69 \%$ and $20.25 \%$ respectively. ${ }^{20,21}$ A 2010 study from Pune reported osteopenic rate of $31.4 \%$ and osteoporosis in $14.3 \%$ of the sample population. ${ }^{22}$

In men, studies reporting the prevalence of osteoporosis are less. In our study, we found the prevalence of osteopenia was $46 \%$ and osteoporosis was $4 \%$. The study by Sharma et al puts the osteoporosis rate at $8.5 \%$ in males over 50 years of age, the difference again attributed to a higher minimum age. ${ }^{9}$ Shetty et al found the prevalence in South Indian males over 50 years at $10.7 \%$ while a similar study by Marwaha et al found the prevalence of osteoporosis in men at $24.6 \% .^{7,23}$

In the present study, the overall prevalence of osteopenia and osteoporosis was $53.2 \%$ and $13.1 \%$ respectively. A study by Vaishya et al determined the overall prevalence of osteoporosis at $9 \%$ in urban northern India. while Marwaha et als study of 1600 patients over 50 years gave an overall value of $35.1 \%$. $^{7,24}$

With such high rates of prevalence and the increased risk of morbidity due to osteoporotic fractures, a screening tool 
is needed which can help identify 'at-risk' patients. The calcaneal QUS has proven to be as effective as DEXA scan in its ability to predict fracture risk. ${ }^{25}$ The QUS has also been reported to give similar results to a central DEXA scan in the elderly. ${ }^{15}$

\section{Limitations}

Our study had certain limitations. The sample size was relatively small and limited to East Delhi. Moreover, other confounding factors like serum calcium levels and vitamin D levels were not assessed. It also had the limitation of being a cross sectional study. A large multicentric longitudinal study would help us better assess the true prevalence of osteoporosis in the country.

\section{CONCLUSION}

The prevalence of decreased bone mass density in our study is high with more than half the sample population being osteopenic or osteoporotic. Osteoporosis is a preventable disease which can as easily be treated. An early diagnosis and treatment can significantly reduce the risk of fractures and other morbidities, thereby reducing the overall global burden of the disease. Even though Indian men over 40 years have lesser prevalence of osteoporosis than women, they are still at risk. Hence, we recommend regular screening of patients to diagnose osteoporosis as well as interventional strategies to raise awareness among the general population.

\section{Funding: No funding sources}

Conflict of interest: None declared

Ethical approval: The study was approved by the institutional ethics committee

\section{REFERENCES}

1. World Health Organization. Assessment of Fracture Risk and Its Application to Screening for Postmenopausal Osteoporosis. Report of a WHO Study Group. Geneva: World Health Organization. 1994.

2. Hunter DJ, Sambrook PN. Bone loss. Epidemiology of bone loss. Arthritis Res Ther. 2000;2:441-5.

3. Riggs BL, Melton LJ 3rd .Evidence for two distinct syndromes of involutional osteoporosis. :The American Journal of Medicine. 1983;75(6):899-901.

4. Warming L, Hassager C, Christiansen C. Changes in bone mineral density with age in men and women: $\mathrm{A}$ longitudinal study. Osteoporos Int. 2002;13:105-12.

5. Mithal A, Kaur P. Osteoporosis in Asia: A Call to Action. Curr Osteoporos Rep. 2012;10:245-7 .

6. Khanna P, Bhargava S. Roentgen assessment of bone density in North Indian population. Indian J Med Res. 1971;59:1599-609.

7. Marwaha RK, Tandon N, Garg MK, Kanwar R, Narang A, Sastry A, et al. Bone health in healthy Indian population aged 50 years and above. Osteoporos Int. 2011;22:2829-36.
8. Cilotti A, Falchetti A. Male osteoporosis and androgenic therapy: From testosterone to SARMs. Clin Cases Miner Bone Metab. 2009;6:229-33.

9. Agrawal NK, Sharma B. Prevalence of osteoporosis in otherwise healthy Indian males aged 50 years and above. Arch Osteoporos. 2013;8:116.

10. Sharma B, Prakash V, Agrawal N, Singh S. Prevalence of male osteoporosis in India. Indian $\mathrm{J}$ Endocrinol Metab. 2012;16:S519.

11. World Health Organization: WHO scientific group on the assessment of osteoporosis at the primary health care level. Summary Meeting Report;Brussels, Belgium. 5-7 May 2004; World Health Organization. 2007.

12. Anburajan M, Ashok KD, Sapthagirivasan V. Vol. 5. Singapore: IACSIT Press; 2011. Evaluation of osteoporosis in Indian women and men using peripheral dual-energy X-ray absorptiometry (pDXA) IPCBE.

13. Malhotra N, Mithal A. Osteoporosis in Indians. Indian J Med Res. 2008;127:263-8.

14. Laugier P. An overview of bone sonometry. Int Congr Ser. 2004;1274:23-32.

15. Bauer DC, Gluer CC, Cauley JA, Vogt TM, Ensrud KE, Genant HK et al. Broadband ultrasound attenuation predicts fractures strongly and independently of densitometry in older women. A prospective study of osteoporotic fractures research group. Arch Intern Med. 1997;157:629-34.

16. Khow KT, Reeve J, Luben R, Bingham S, Welch A, Wareham $\mathrm{N}$ et al. Prediction of total hip fracture risk in men and women by quantative ultrasound of the calcaneus: EPIC-Norfolk prospective population study. Lancet. 2004;363:197-202.

17. Diez-Perez A, Marin F, Vila J, Abizanda M, Cervera $\mathrm{A}$, Carbonell $\mathrm{C}$, et al. Evaluation of calcaneal quantitative ultrasound in a primary care setting as a screening tool for osteoporosis in postmenopausal women. J Clin Densitom. 2003;6:237-45.

18. Knapp KM, Blake GM, Spector TD, Fogelman I. Can the WHO definition of osteoporosis be applied to multi-site axial transmission quantitative ultrasound? Osteoporos Int. 2004;15:367-74.

19. Jin NN, Lin SQ, Zhang Y, Chen FL. Assess the discrimination of Achilles InSight calcaneus quantitative ultrasound device for osteoporosis in Chinese women: Compared with dual energy X-ray absorptiometry measurements. Eur $\mathrm{J}$ Radiol. 2010;76:265-8.

20. Gandhi AB, Shukla AK. Evaluation of BMD of women above 40 years of age. J Obstet Gynecol India. 2005;55(3):265-7.

21. Sharma S, Tandon VR, Mahajan A, Kour A, Kumar D. Preliminary screening of osteoporosis and osteopenia in urban women from Jammu using calcaneal QUS. Indian J Med Sci. 2006;60(5):183-9.

22. Unni J, Garg R, Pawar R. Bone mineral density in women above 40 years. J Midlife Health. 2010;1(1):19-22. 
23. Shetty S, Kapoor N, Naik D, Asha HS, Prabu S, Thomas N, et al. Osteoporosis in healthy South Indian males and the influence of life style factors and vitamin d status on bone mineral density. J Osteoporos. 2014;2014:723238

24. Vaishya R, Vijay V, Agarwal AK, Maheshwari P. Assessment of osteoporotic fracture risk in urban Indian population using quantitative ultrasonography \& FRAX tool. Indian J Med Res. 2017;146(8):51-6.
25. Baran DT, Kelly AM, Karellas A, Gionet M, Price M, Leahey D, Steuterman S, McSherry B, Roche J. Ultrasound attenuation of the os calcis in women with osteoporosis and hip fractures. Calcif Tissue Int. 1988;43(3):138-42.

Cite this article as: Sharma A, Garg A, Singh B. The prevalence of osteopenia and osteoporosis in out-patients above 40 years of a tertiary care hospital in Delhi. Int J Res Orthop 2022;8:222-6. 